11,19

\title{
Тепловые, оптические и диэлектрические свойства $\mathrm{Rb}_{2} \mathrm{TaF}_{7}$
}

\author{
(C) Е.И. Погорельцев ${ }^{1,2}$, С.В. Мельникова ${ }^{1}$, А.В. Карташев ${ }^{1,3}$, \\ М.В. Горев ${ }^{1,2}$, И.Н. Флёров ${ }^{1,2}$, Н.М. Лапташ ${ }^{4}$ \\ ${ }^{1}$ Институт ффизики им. Л.В. Киренского СО РАН, \\ Красноярск, Россия \\ ${ }^{2}$ Институт инженерной физики и радиоэлектроники Сибирского федерального университета, \\ Красноярск, Россия \\ ${ }^{3}$ Красноярский педагогический университет им. В.П. Астафьева, \\ Красноярск, Россия \\ ${ }^{4}$ Институт химии ДВО РАН, \\ Владивосток, Россия \\ E-mail: pepel@iph.krasn.ru \\ (Поступила в Редакцию 26 сентября 2016 г. \\ В окончательной редакции 19 октября 2016 г.)
}

Выполнены исследования теплофизических, оптических и диэлектрических свойств $\mathrm{Rb}_{2} \mathrm{TaF}_{7}$. Изменение химического давления во фторидах $A_{2}^{+} \mathrm{TaF}_{7}$ за счет катионного замещения аммония рубидием не повлияло на сегнетоэластическую природу структурных искажений, но привело к стабилизации высоко- и низкотемпературной фаз и к росту двулучепреломления. Энтропия фазового перехода $P 4 / n m m \leftrightarrow C m m a$ характерна для превращений типа смещения, что согласуется с моделью структур исходной и искаженной фаз. Анизотропия химического давления является причиной изменения знаков аномальной деформации и барического коэффициента $d T / d p$ для $\mathrm{Rb}_{2} \mathrm{TaF}_{7}$ по сравнению с наблюдаемыми для аммонийного аналога.

Исследование выполнено при финансовой поддержке РФФИ и правительства Красноярского края в рамках научного проекта № 16-42-243001.

DOI: 10.21883/FTT.2017.05.44387.358

\section{1. Введение}

Среди множества неорганических фторидов и оксифторидов встречаются соединения со всевозможными структурными модификациями и связанными с ними разнообразными функциональными свойствами и эффектами, которые находят практическое применение в развитии различных технологий, таких, например, как производство и хранение энергии, фотоника, микроэлектроника и др. [1,2]. Как правило, речь идет об использовании физических свойств, имеющих место при комнатной температуре и атмосферном давлении. Именно поэтому до недавнего времени главное направление исследований неорганических фторсодержащих материалов в основном было связано с синтезом новых соединений и их структурной характеризацией. Однако известно, что физические свойства могут демонстрировать аномальное изменение при трансформации структуры в результате фазовых переходов как под влиянием внешних воздействий в виде изменения температуры, давления, электрического и магнитного полей, так и за счет изменения химического давления, вызванного катион/анионными замещениями $[3,4]$. В настоящее время поиск путей целенаправленного варьирования химического состава, структуры и физических свойств материалов, в том числе фторсодержащих, рассматривается в качестве одного из перспективных направлений исследований. При этом необходимо иметь в виду, что значительную роль в формировании структуры, ее потенциальной трансформации и физических свойств играют состав и форма фторных и фторкислородных полиэдров $\left[\mathrm{MeX}_{n}\right](M e-$ центральный атом, $X-\mathrm{O}, \mathrm{F}$, $n=4-9)$, являющихся одними из главных структурных элементов кристаллов [1-4]. Полиэдры с $n=4$ и 5 встречаются редко. Наиболее распространены структурные элементы $\left[\mathrm{MeX}_{6}\right],\left[\mathrm{MeX}_{7}\right],\left[\mathrm{MeX}_{8}\right]$ и $\left[\mathrm{MeX}_{9}\right]$ [2]. В большей степени изучены фазовые переходы во фторидах и оксифторидах с октаэдрическими анионами [3-5]. С другой стороны, несомненный интерес в качестве центральных атомов $M е$ представляют металлы, которые в зависимости от валентности способны в совокупности с фторными и фторкислородными лигандами $X$ образовывать полиэдры различной конфигурации. К таковым, в частности, относятся $\mathrm{Zr}, \mathrm{Hf}, \mathrm{Nb}$, Та, для которых известны шести-, семи- и восьмикоординированные анионные полиэдры. Несмотря на сравнительно широкий круг публикаций, посвященных синтезу и исследованию такого рода соединений [6-15], отсутствует систематическое изучение влияния структурных особенностей на термодинамическую устойчивость кристаллических фаз и физические свойства.

В настоящей работе выполнены измерения теплоемкости, теплового расширения, оптического двойникования, двулучепреломления и диэлектрической проницаемости фторида $\mathrm{Rb}_{2} \mathrm{TaF}_{7}$, являющиеся логическим продолжением исследований фазовых переходов в со- 
единениях ряда $\mathrm{A}_{2} \mathrm{MeF}_{7}$ с семикоординированным анионным полиэдром, начатых в $[7,15,16]$. Симметрия кристаллической решетки этих фторидов при комнатной температуре зависит от ионного радиуса одновалентного катиона: для $A=\mathrm{Rb}$ и $\mathrm{NH}_{4}-$ тетрагональная $(P 4 / n m m, Z=2)[15,16]$, в случае $A=\mathrm{K}-$ моноклинная (пр. гр. $P 2_{1} / c, Z=4$ ) [7]. Семикоординированный анионный полиэдр $\left[\mathrm{MeFe}_{7}\right]$ имеет полярную ромбическую симметрию и представляет собой моношапочную тригональную призму (monocapped trigonal prism). Сведения о термодинамической устойчивости фазы $P 22_{1} / c$ в кристалле $\mathrm{K}_{2} \mathrm{TaF}_{7}$ отсутствуют. Информация о наличии в $\mathrm{Rb}_{2} \mathrm{TaF}_{7}$ одного структурного превращения в районе $145 \mathrm{~K}$ и о симметрии исходной $(P 4 / \mathrm{nmm})$ и искаженной (Стта, $Z=4)$ фаз представлена в [15]. В аммонийном соединении обнаружены и достаточно подробно исследованы два последовательных структурных превращения $G_{1}(P 4 / n m m) \leftrightarrow G_{2}(P m m n) \leftrightarrow G_{3}$ (тетрагональная фаза) [16]. Таким образом, катионное замещение $\mathrm{K} \rightarrow \mathrm{Tb} \rightarrow \mathrm{NH}_{4}$ существенно влияет на симметрию кристаллов $\mathrm{A}_{2} \mathrm{MeF}_{7}$ и последовательность фазовых переходов. Именно поэтому несомненный интерес представляют исследования физических свойств фторида $\mathrm{Rb}_{2} \mathrm{TaF}_{7}$, которые позволят установить природу и механизм искажений структуры.

\section{2. Экспериментальные результаты}

В настоящей работе исследования выполнены на кристаллах $\mathrm{Rb}_{2} \mathrm{TaF}_{7}$, использовавшихся при изучении структуры в [15], где описана также процедура синтеза.

Калориметрические исследования выполнены в два этапа. На первой стадии был проведен поиск фазовых переходов в $\mathrm{Rb}_{2} \mathrm{TaF}_{7}$ в более широком интервале температур, чем в [15], где был обнаружен обратимый фазовый

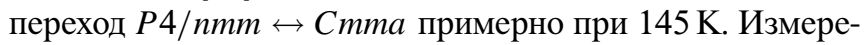
ния выполнены с помощью дифференциального сканирующего микрокалориметра ДСМ-10М (ДСМ) на образце массой $\sim 0.1 \mathrm{~g}$ в диапазоне температур от 100 до $400 \mathrm{~K}$ в режимах нагрева и охлаждения со скоростью $8 \mathrm{~K} \cdot \mathrm{min}^{-1}$. Результаты исследований подтверждают наличие одного фазового перехода при $T_{0}=150 \pm 2 \mathrm{~K}$. Определены интегральные параметры фазового перехода (изменения энтальпии и энтропии) путем интегрирования соответствующих функций: $\Delta H=\int \Delta C_{p} d T=275 \mathrm{~J} \cdot \mathrm{mol}^{-1}$, $\Delta S=\int\left(\Delta C_{p} / T\right) d T=1.8 \mathrm{~J} \cdot(\mathrm{mol} \cdot \mathrm{K})^{-1}$.

На втором этапе проведены прецизионные калориметрические исследования на адиабатическом калориметре, конструкция которого и методика измерения теплоемкости описаны в [17]. Измерения в интервале температур $78-300 \mathrm{~K}$ проводились на образце массой $0.384 \mathrm{~g}$, приготовленном в виде цилиндрической таблетки $(d=8 \mathrm{~mm}$, $h=2 \mathrm{~mm}$ ), спрессованной из порошка, полученного путем перетирания мелких монокристаллов.

На рис. 1 показана молярная теплоемкость кристалла $\mathrm{Rb}_{2} \mathrm{TaF}_{7}$.

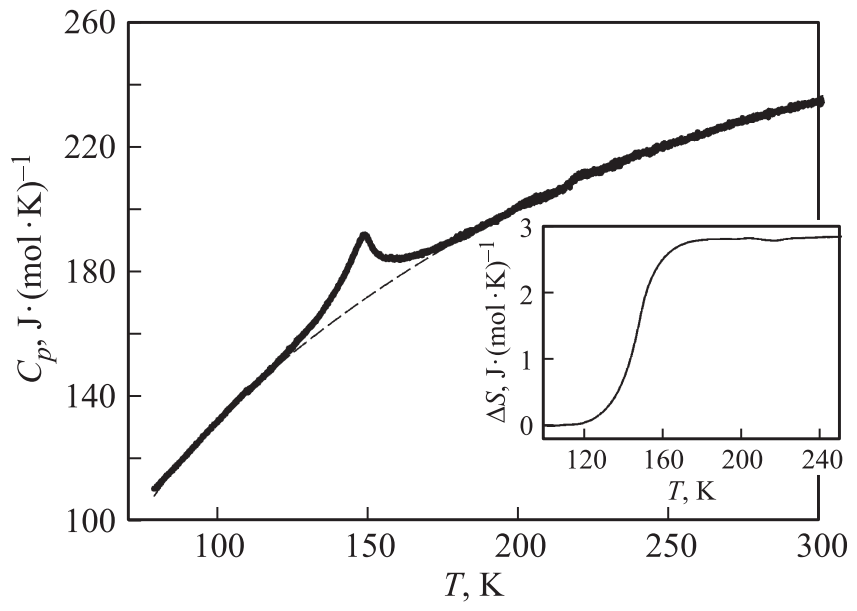

Рис. 1. Температурные зависимости молярной теплоемкости $\mathrm{Rb}_{2} \mathrm{TaF}_{7}$ в широкой области температур и аномальной энтропии, связанной с фазовым переходом (на вставке). Штриховая линия - решеточная теплоемкость.

Обнаружена одна аномалия в виде пика с максимумом при температуре $T_{0}=147.9 \pm 0.5 \mathrm{~K}$, принятой за температуру структурного превращения. Штриховой линией в области фазового перехода показана решеточная теплоемкость, полученная путем интерполяции данных $C_{p}(T)$, удаленных от перехода выше и ниже $T_{0}$, комбинацией функций Дебая и Эйнштейна. В результате были уточнены значения энтальпии и энтропии фазового перехода, которые составили $\Delta H=390 \pm 30 \mathrm{~J} \cdot \mathrm{mol}^{-1}$ и $\Delta S=2.6 \pm 0.2 \mathrm{~J} \cdot(\mathrm{mol} \cdot \mathrm{K})^{-1}$ соответственно. Оба интегральных параметра оказались больше, чем определенные методом ДСМ, что связано с более высокой чувствительностью адиабатического калориметра.

Дилатометрические исследования проводились на образце, использованном при калориметрических измерениях. Эксперименты по определению температурного поведения линейной деформации $\Delta L / L$ и коэффициента линейного теплового расширения $\alpha$ выполнены на индукционном дилатометре DIL-402C фирмы NETZSCH в потоке сухого газообразного гелия. Скорости нагрева образца в интервале температур $105-400 \mathrm{~K}$ варьировались от 2 до $4 \mathrm{~K} \cdot \mathrm{min}^{-1}$. Эталон из плавленого кварца был использован для калибровки и учета расширения измерительной системы. Данные, полученные в нескольких сериях измерений, согласовывались между собой в пределах 2-3\%.

На рис. 2 представлены температурные зависимости $\Delta L / L(T)$ и $\alpha(T)$, измеренные в режиме нагрева.

Как и в калориметрических экспериментах наблюдалась одна аномалия. Температура максимума пика $\alpha(T)$ $T_{0}=145 \pm 1 \mathrm{~K}$, принятая за температуру фазового перехода, удовлетворительно согласуется с величиной $T_{0}$, определенной в измерениях теплоемкости. На вставке к pис. 2, $a$ показано поведение аномальной части линейной деформации $(\Delta L / L)_{\text {an. }}$ Видно, что изменение длины 

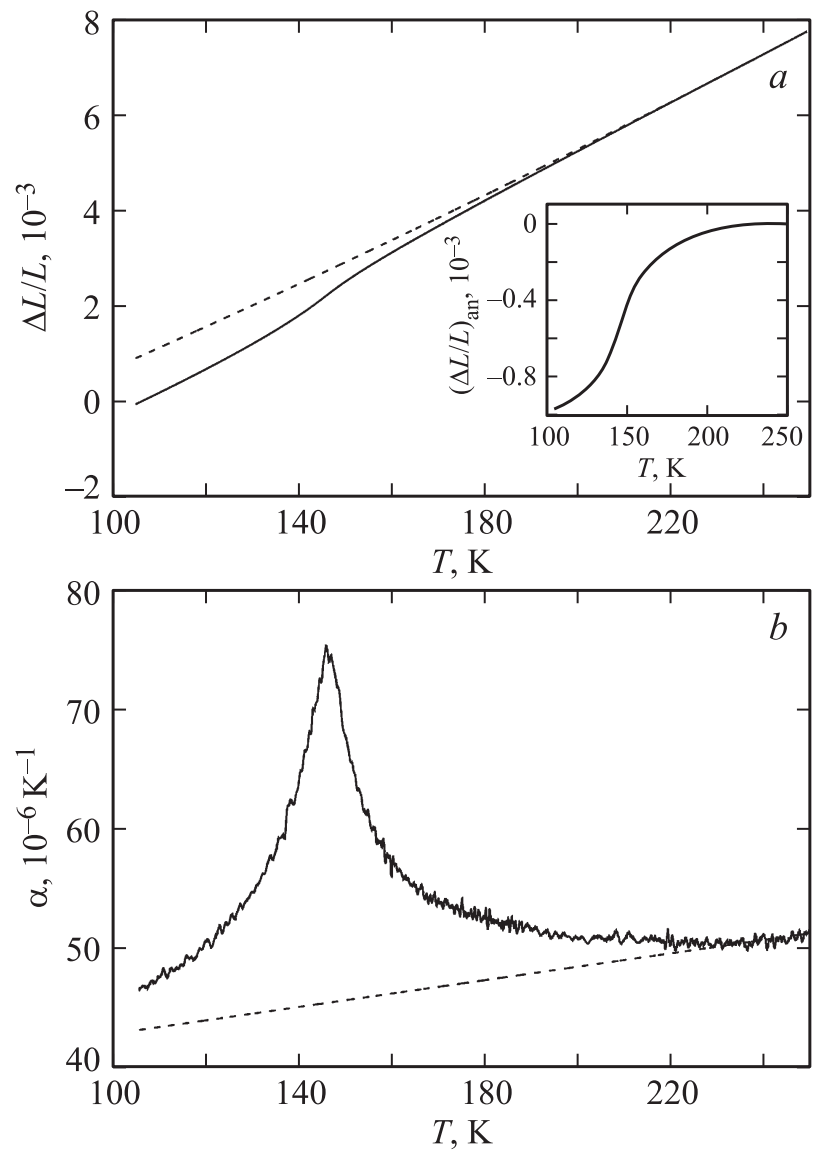

Pис. 2. Температурные зависимости линейной деформации $(a)$ и ее аномальной составляющей (на вставке), а также коэффициента линейного теплового расширения $(b)$. Штриховая линия - решеточный вклад.

образца, связанное с фазовым переходом, наблюдается вплоть до $100 \mathrm{~K}$ и составляет $\sim 0.1 \%$.

Поляризационно-оптические исследования и измерения двулучепреломления кристалла $\mathrm{Rb}_{2} \mathrm{TaF}_{7}$ проведены на очень тонких $(d=43 \mu \mathrm{m})$ пластинках роста с ориентацией $(001)_{T}$ при помощи микроскопа „Axioskop40“ и температурной камеры „Linkam LTS 350“. Точность измерений абсолютных значений двулучепреломления $\Delta n_{c}$, выполненных методом компенсатора Берека (Leica) на тонкой пластинке, не высока: \pm 0.0001 , тогда как чувствительность к изменениям $\Delta n_{c}(T)$ составляет \pm 0.00001 .

При комнатной температуре образец оптически изотропен в соответствии с тетрагональной симметрией. В процессе охлаждения вблизи $T_{0} \sim 140 \mathrm{~K}$ в поле зрения микроскопа появляются оптическая анизотропия и очень мелкая полосчатая двойниковая структура с границами вдоль [100]. Общее погасание пластинки происходит по [110] (рис. 3) и не изменяется при дальнейшем понижении температуры. Таким образом, при фазовом переходе сингония кристалла понижается от тетрагональной до ромбической. Геометрия экспе- римента указывает на потерю кристаллом элементов симметрии типа $(100)_{T}$ и формирование осей элементарной ячейки низкотемпературной фазы вдоль $[110]_{R}$ (базоцентрированная ячейка $C$ ). Таким образом, наши наблюдения подтверждают верность сделанных в [15] выводов об изменении симметрии при фазовом переходе в кристалле $\mathrm{Rb}_{2} \mathrm{TaF}_{7}: G_{2}(C m m a) \leftrightarrow G_{1}(P 4 / n m m)$. Картина двойникования в рубидиевом соединении радикально отличается от наблюдавшейся ранее в $\left(\mathrm{NH}_{4}\right)_{2} \mathrm{TaF}_{7}$ [16], где в ромбической фазе элементарная ячейка остается примитивной: $G_{1}(P 4 / n m m) \leftrightarrow G_{2}(P m m n) \leftrightarrow G_{3}$ (тетрагональная фаза).

Измерения двулучепреломления выполнены в квазистатическом режиме в процессах нагрева и охлаждения. Температурная зависимость $\Delta n_{c}(T)$ представлена на рис. 4. Двулучепреломление скачком появляется

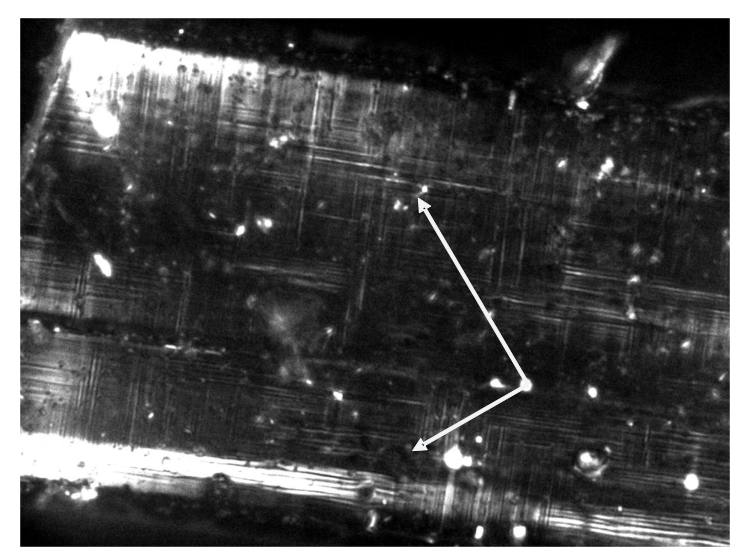

Рис. 3. Наблюдение двойникования низкотемпературной фазы в пластинках роста $(001)_{T}$ кристалла $\mathrm{Rb}_{2} \mathrm{TaF}_{7}$.

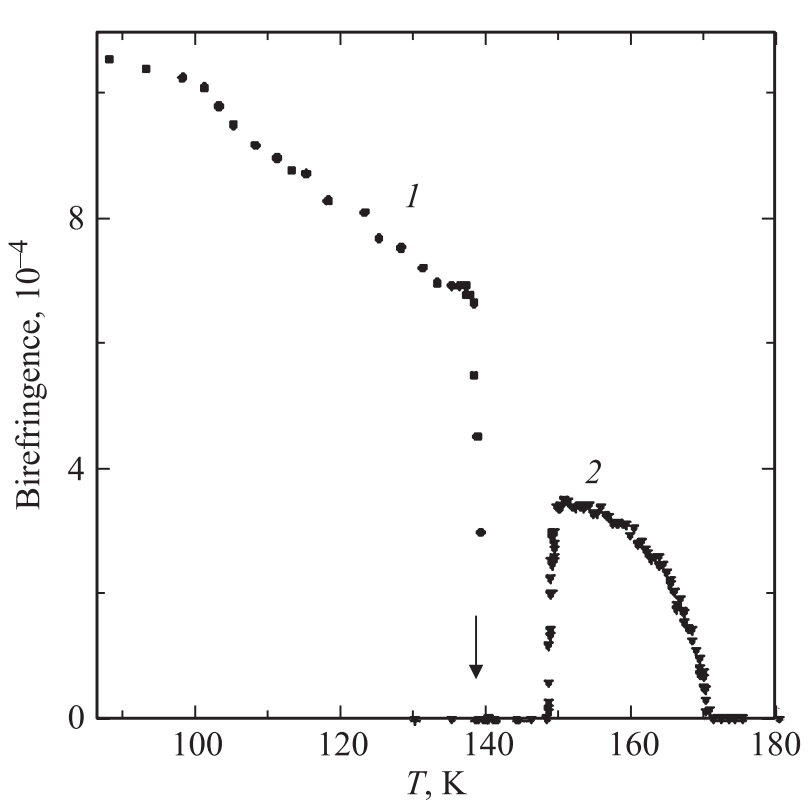

Рис. 4. Температурные зависимости двулучепреломления $\Delta n_{c}(T)$ в кристаллах $\mathrm{Rb}_{2} \mathrm{TaF}_{7}(1)$ и $\left(\mathrm{NH}_{4}\right)_{2} \mathrm{TaF}_{7}[16]$ (2). 


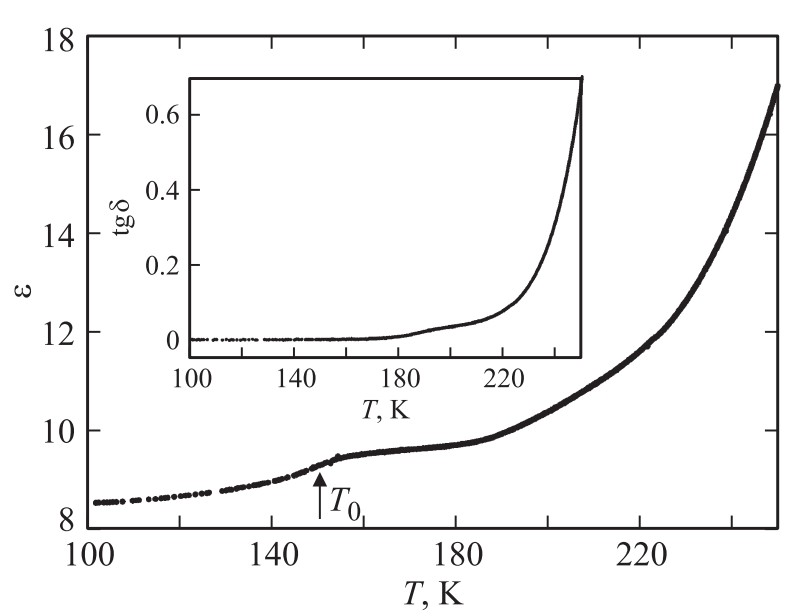

Рис. 5. Температурные зависимости диэлектрической проницаемости $\varepsilon$ и тангенса угла диэлектрических потерь $\operatorname{tg} \delta$ (на вставке) $\mathrm{Rb}_{2} \mathrm{TaF}_{7}$, измеренные на частоте $1 \mathrm{kHz}$.

в процессе охлаждения и исчезает при нагреве при температуре $T_{0}=138-139 \mathrm{~K}$. Для сравнения здесь же представлено двулучепреломление в родственном соединении $\left(\mathrm{NH}_{4}\right)_{2} \mathrm{TaF}_{7}$ [16]. Видно, что замещение аммония рубидием привело к существенному понижению температуры фазового перехода между тетрагональной и ромбической фазами, а также к небольшому увеличению двулучепреломления. Кроме того, переход в ромбическую фазу рубидиевого соединения имеет явные черты перехода первого рода, о чем свидетельствуют скачок $\Delta n_{c}(T)$ и небольшой температурный гистерезис $\delta T_{0}=0.5 \mathrm{~K}$. В аммонийном соединении переход $G_{1}(P 4 / n m m) \leftrightarrow G_{2}($ Pmmn $)$ относится к переходам второго рода. Обнаруженные особенности поведения оптических свойств $\mathrm{Rb}_{2} \mathrm{TaF}_{7}$ по сравнению с аналогичными свойствами $\left(\mathrm{NH}_{4}\right)_{2} \mathrm{TaF}_{7}$, вероятнее всего, обусловлены различной симметрией ромбической фазы кристаллов.

Несмотря на то что катионное замещение не сопровождалось изменением сингонии фазы ниже $T_{0}$, которая к тому же осталась сегнетоэластической, нами проведены исследования диэлектрической проницаемости. Измерения проводились на псевдокерамическом образце, использованном при исследовании тепловых свойств. Серебряные электроды наносились методом вакуумного напыления. Возможность исследования диэлектрических свойств такого рода образцов продемонстрирована путем сравнения данных о зависимостях $\varepsilon(T)$, полученных на монокристаллических пластинках и псевдокерамических образцах оксифторидов [18].

На рис. 5 показана температурная зависимость диэлектрической проницаемости, на которой в области $140-160 \mathrm{~K}$ наблюдается аномальное поведение в виде небольшой размытой ступеньки. Такое поведение $\varepsilon$ указывает на то, что соединение $\mathrm{Rb}_{2} \mathrm{TaF}_{7}$ испытывает фазовый переход несегнетоэлектрической природы. Точка перегиба функции $\varepsilon(T)$ находится вблизи $150 \mathrm{~K}$, что удо- влетворительно совпадает с температурой структурного превращения, установленной в других экспериментах. На зависимости тангенса угла диэлектрических потерь (вставка на рис. 5) также присутствует бугрообразная аномалия при температуре $\sim 190 \mathrm{~K}$, т.е. существенно выше $T_{0}$. Различие в температурах особенностей на зависимостях $\varepsilon(T)$ и $\operatorname{tg} \delta(T)$ объясняется, скорее всего, тем, что в изменение емкости образца вносит вклад изменение его линейных размеров при фазовом переходе, в то время как диэлектрические потери связаны, в частности, с процессами релаксации. Такое же различие в особых температурах наблюдалось и при исследовании $\left(\mathrm{NH}_{4}\right)_{2} \mathrm{TaF}_{7}$ [16].

\section{3. Обсуждение результатов}

Комплексные исследования ряда физических свойств в интервале температур $78-400 \mathrm{~K}$ показали, что $\mathrm{Rb}_{2} \mathrm{TaF}_{7}$ не испытывает фазовых переходов, кроме $P 4 / n m m \rightarrow C m m a$, обнаруженного в [15]. Поведение двойникования, двулучепреломления и диэлектрической проницаемости этого фторида аналогично поведению соответствующих характеристик $\left(\mathrm{NH}_{4}\right)_{2} \mathrm{TaF}_{7}$, установленных в [16], что свидетельствует о сохранении сегнетоэластического состояния в ромбической фазе $\mathrm{Rb}_{2} \mathrm{TaF}_{7}$, несмотря на смену примитивной ячейки на базоцентрированную. В то же время катионное замещение аммония рубидием приводит к расширению температурной области стабильности исходной тетрагональной фазы за счет понижения температуры структурного превращения.

Небольшая величина энтропии $\left(\Delta S_{0} \approx 0.3 R \ll R \ln _{2}\right)$ свидетельствует об отсутствии ярко выраженных процессов упорядочения структурных элементов при фазовом переходе в $\mathrm{Rb}_{2} \mathrm{TaF}_{7}$. В аммонийном фториде полное изменение энтропии, связанное с двумя последовательными структурными превращениями, было больше: $\Sigma \Delta S=\Delta S_{1}+\Delta S_{2} \approx 0.7 R \approx R \ln 2$ [16]. Из поведения теплоемкости и теплового расширения $\left(\mathrm{NH}_{4}\right)_{2} \mathrm{TaF}_{7}$ следует, что аномальные вклады, связанные с ярко выраженным переходом первого рода между ромбической Pmmn и низкотемпературной тетрагональной фазами, накладываются на ниспадающие аномальные ветви $\Delta C_{p}(T)$ и $\Delta \alpha(T)$ ниже $T_{1}$. Вычитая из $\Sigma \Delta S$ изменение энтропии, обусловленное структурным превращением при $T_{2}\left(\Delta S_{2} \approx 0.2 R\right)$, получаем, что энтропия перехода $P 4 / n m m \leftrightarrow P$ Рmп составляет $\Delta S_{1} \approx 0.5 R$.

Чтобы дискутировать о механизме искажений структуры в результате фазовых переходов, необходим совместный анализ особенностей энтропийных параметров и структурных данных о тепловых параметрах колебаний критических атомов и/или ионных групп в исходной и искаженной фазах. Для $\left(\mathrm{NH}_{4}\right)_{2} \mathrm{TaF}_{7}$ подробные сведения о структуре отсутствуют. Модели структур фаз $P 4 / n m m$ и Cmma в $\mathrm{Rb}_{2} \mathrm{TaF}_{7}$ были предложены на основе монокристальных рентгеновских исследований [15]. Координационный анионный полиэдр [ $\left.\mathrm{TaF}_{7}\right]$ 
представляет собой моношапочную тригональную призму. В фазе $P 4 / n m m$ атом F1, лежащий на оси четвертого порядка, и четыре экваториальных атома F2, лежащие в плоскости, перпендикулярной этой оси, упорядочены. Два атома F3 динамически разупорядочены по четырем кристаллографическим позициям при повороте вокруг оси $c$. Таким образом, семикоординированный полиэдр $\left[\mathrm{TaF}_{7}\right]$ разупорядочен и имеет две ориентации относительно локальной оси четвертого порядка. В случае полного упорядочения полиэдров (атомов фтора) в фазе Cmma изменение энтропии могло бы составить $\Delta S_{0}=0.71 R=R \ln 2$. Однако в [15] установлено, что после перехода фторный полиэдр остается разупорядоченным, но одновременно наблюдается значительное уменьшение тепловых параметров атомов фтора. Это обстоятельство вполне удовлетворительно согласуется с небольшой величиной энтропии перехода $\Delta S_{0} \approx 0.3 R \ll R \ln 2$, которая пропорциональна параметру ангармоничности $\delta=\langle\bar{x}\rangle^{2} / a^{2}$, связывающему амплитуду колебаний критического атома/иона $\bar{x}$ со средним межатомным расстоянием $a$ [19].

Следует отметить, что колебания одного из двух неэквивалентных атомов $\mathrm{Rb}$ скоординированы с движением разупорядоченных атомов фтора и, таким образом, тоже являются ярко выраженными анизотропными. С большой уверенностью можно полагать, что во фториде $\left(\mathrm{NH}_{4}\right)_{2} \mathrm{TaF}_{7}$ колебания тетраэдрического иона $\mathrm{NH}_{4}^{+}$, занимающего соответствующую кристаллографическую позицию, могут быть в большей степени ангармоническими, чем характерные для сферического атома $\mathrm{Rb}$, и в результате перехода при $T_{1}$ существует дополнительный вклад в энтропию. Очевидно также, что низкотемпературный переход в тетрагональную фазу в $\left(\mathrm{NH}_{4}\right)_{2} \mathrm{TaF}_{7}$, отсутствующий в $\mathrm{Rb}_{2} \mathrm{TaF}_{7}$, скорее всего, может быть связан с изменением движения аммонийного катиона.

Абсолютные значения коэффициентов линейного теплового расширения кристаллов $\mathrm{Rb}_{2} \mathrm{TaF}_{7}$ и $\left(\mathrm{NH}_{4}\right)_{2} \mathrm{TaF}_{7}$ [16] практически совпадают вдали от температур фазовых превращений $(T=240 \mathrm{~K}$, $\left.\alpha \approx 5 \cdot 10^{-5} \mathrm{~K}^{-1}\right)$. Однако в области перехода между тетрагональной $P 4 / n m m$ и ромбическими (Cmma, Pmmn) фазами аномальное поведение $\Delta L / L$ и $\alpha$ фторидов значительно различается. В аммонийном кристалле аномальный вклад в коэффициент объемного теплового расширения, рассматриваемый для керамического образца как $\beta=3 \alpha$, характеризуется отрицательным знаком, т. е. $\Delta \beta=\beta-\beta_{\text {lat }}<0$ ( $\beta_{\text {lat }}-$ решеточный вклад) $[16]$. В соответствии с этим в рамках уравнения Эренфеста [20] барический коэффициент, характеризующий сдвиг температуры перехода $T_{1}$ под давлением, тоже является отрицательным $d T_{1} / d p=T_{1}\left(\Delta \beta / \Delta C_{p}\right)=-110 \mathrm{~K} \cdot \mathrm{GPa}^{-1}$. Для рубидиевого фторида $\Delta \beta>0$, как следует из рис. 2, $b$, что приводит к повышению температуры перехода под давлением. Расчет с помощью уравнения Эренфеста с учетом данных о скачках теплоемкости $\Delta C_{p}=21 \mathrm{~J} \cdot(\mathrm{mol} \cdot \mathrm{K})^{-1}$ и коэффициента объемного расширения $\Delta \beta=75 \mathrm{~K}^{-1}$ дает для $\mathrm{Rb}_{2} \mathrm{TaF}_{7}$ $d T_{0} / d p=57 \mathrm{~K} \cdot \mathrm{GPa}^{-1}$. Анализ зависимостей коэффициента теплового расширения и теплоемкости в рамках уравнения Пиппарда позволил установить линейную зависимость $\beta(T)$ от $C_{p}(T)$ в интервале $\sim\left(T_{0}-6\right) \mathrm{K}$, в соответствии с которой барический коэффициент составляет $d T_{0} / d p=32 \mathrm{~K} \cdot \mathrm{GPa}^{-1}$. Довольно существенное различие значений $d T_{0} / d p$, определенных для $\mathrm{Rb}_{2} \mathrm{TaF}_{7}$ из сопоставления аномальных величин $\Delta \beta$ и $\Delta C_{p}$ в точке перехода и из зависимостей полных величин $\beta(T)$ и $C_{p}(T)$, может объясняться разной степенью размытия соответствующих функций вследствие проведения экспериментов на квазикерамических образцах с различными скоростями изменения температуры в процессах измерения теплоемкости и теплового расширения.

Причину разного знака барических коэффициентов при фазовом переходе $P 4 / n m m \leftrightarrow(C m m a, P m m n)$ в $\mathrm{Rb}_{2} \mathrm{TaF}_{7}$ и $\left(\mathrm{NH}_{4}\right)_{2} \mathrm{TaF}_{7}$ можно объяснить, рассматривая изменение линейных и объемных параметров элементарной ячейки в результате катионного замещения аммония рубидием. Несмотря на то что ионный радиус атома $\mathrm{Rb}$ больше, чем у катионной группы $\mathrm{NH}_{4}$ (независимо от числа ближайших соседей), объем элементарной ячейки P4/nmm $\mathrm{Rb}_{2} \mathrm{TaF}_{7}$ (371.06 $\left.\AA^{3}[15]\right)$ оказался меньше по сравнению с $\left(\mathrm{NH}_{4}\right)_{2} \mathrm{TaF}_{7}\left(371.30 \AA^{3}[16]\right)$. Объясняется это тем, что изменение химического давления за счет замещения аммония рубидием оказалось анизотропным, о чем свидетельствуют увеличение параметров $a$ и $b$ на $0.29 \%$ и уменьшение параметра $c$ на $0.64 \%$, что и привело к уменьшению объема.

\section{4. Заключение}

В результате исследования теплофизических, оптических и диэлектрических свойств $\mathrm{Rb}_{2} \mathrm{TaF}_{7}$ и сравнения их с ранее изученными для $\left(\mathrm{NH}_{4}\right)_{2} \mathrm{TaF}_{7}[16]$ установлено, что замещение тетраэдрического катиона аммония сферическим рубидием привело к следующим обстоятельствам.

Фазовый переход $P 4 / n m m \leftrightarrow C m m a$ в $\mathrm{Rb}_{2} \mathrm{TaF}_{7}$, обнаруженный в [15], является сегнетоэластическим, не связанным с возникновением сегнетоэлектрического состояния.

В соответствии с энтропией фазового перехода $\Delta S_{0} \approx 0.3 R$ ярко выраженные процессы упорядочения структурных элементов отсутствуют, что удовлетворительно согласуется с моделью структур исходной и искаженной фаз.

Изменение химического давления за счет катионного замешения оказалось анизотропным, приводящим к увеличению параметров $a$ и $b$ и значительному уменьшению параметра $c$. Это в свою очередь вызвало изменение знаков аномального коэффициента теплового расширения и барического коэффициента $d T / d p$ : в отличие от $\left(\mathrm{NH}_{4}\right)_{2} \mathrm{TaF}_{7}$, для которого $d T / d p<0$, температура фазового перехода в $\mathrm{Rb}_{2} \mathrm{TaF}_{7}$ растет с ростом гидростатического давления. 


\section{Список литературы}

[1] A. Tressaud. Functionalized inorganic fluorides. WileyBlackwell, Chichester (2010). 591 p.

[2] M. Leblanc, V. Maisonneuve, A. Tressaud. Chem. Rev. 115, 1191 (2015).

[3] I. Flerov, M. Gorev, K. Aleksandrov, A. Tressaud, J. Grannec, M. Couzi. Mater. Sci. Eng. R 24, 81 (1998).

[4] I.N. Flerov, M.V. Gorev, M.S. Molokeev, N.M. Laptash. In: Photonic and electronic properties of fluoride materials / Eds by A. Tressaud, K. Poeppelmeier. Elsevier (2016). Ch. 16.

[5] I.N. Flerov, M.V. Gorev, A. Tressaud, N.M. Laptash. Cryst. Rep. 56, 9 (2011).

[6] E.C. Reynhardt, J.C. Pratt, A. Watton, H.E. Petch. J. Phys. C 14, 4701 (1981).

[7] R.B. English, A.M. Heyns, E.C. Reynhardt. J. Phys. C 16, 829 (1983).

[8] L.-S. Du, R.W. Schurko, K.H. Lim, C.P. Grey. J. Phys. Chem. A 105, 760 (2001).

[9] V.D. Fokina, M.V. Gorev, E.V. Bogdanov, E.I. Pogoreltsev, I.N. Flerov, N.M. Laptash. J. Fluor. Chem. 154, 1 (2013).

[10] A.M. Rodriguez, M.C. Caracoche, J.A. Martinez, A.R. Lopez Garcia. Hyperfine Interact. 30, 277 (1986).

[11] В.Д. Фокина, И.Н. Флёров, М.В. Горев, Е.В. Богданов, А.Ф. Бовина, Н.М. Лапташ. ФТТ 49, 1475 (2007).

[12] В.Д. Фокина, А.Ф. Бовина, Е.В. Богданов, Е.И. Погорельцев, Н.М. Лапташ, М.В. Горев, И.Н. Флёров. ФТТ 53, 2038 (2011).

[13] I.N. Flerov, I.N. Gorev, V.D. Fokina, A.F. Bovina, E.V. Bogdanov, E.I. Pogoreltsev, N.M. Laptash. J. Fluor. Chem. 132, 713 (2011).

[14] W. Weber, E. Schweda. Mater. Sci. Forum. 228, 353 (1996).

[15] N.M. Laptash, A.A. Udovenko, T.B. Emelina. J. Fluor. Chem. 132, 1152 (2011).

[16] Е.И. Погорельцев, С.В. Мельникова, А.В. Карташев, М.С. Молокеев, М.В. Горев, И.Н. Флёров, Н.М. Лапташ. ФTT 55, 555 (2013).

[17] В.С. Бондарев, А.В. Карташев, А.Г. Козлов, И.Я. Макиевский, И.Н. Флёров, М.В. Горев. Автоматизация калориметрических установок. Препринт № 829Ф. ИФ СО РАН, Красноярск (2005). 40 с.

[18] И.Н. Флёров, В.Д. Фокина, А.Ф. Бовина, Е.В. Богданов, М.С. Молокеев, А.Г. Кочарова, Е.И. Погорельцев, Н.М. Лапташ. ФТТ 50, 497 (2008).

[19] В.Г. Вакс. Введение в микроскопическую теорию сегнетоэлектриков. Наука, М. (1973), 327 с.

[20] Л.Д. Ландау, Е.М. Лифшиц. Статистическая физика. Наука, М. (1964). 568 c. 